\title{
Key Technology of Monitoring and Control for Coal Shearer Supporting Network Convergence
}

\section{Quanzhu Zhang}

North China Institute of Science and Technology, Beijing 065201, China

E-mail: zhangquanzhu1965@126.com

\section{$\mathrm{Na} \mathrm{Ma}{ }^{12}$}

North China Institute of Science and Technology, Beijing 065201, China

E-mail: 84432379@qq.com

\section{Chengyu Huang}

North China Institute of Science and Technology, Beijing 065201, China

In order to solve disadvantages of the existing monitoring system for coal shearer, such as complex wiring and maintenance difficulties as well as high-cost, a low cost and easilyequipped wireless network was designed. The CC2530 was used as the core unit to build the ZigBee network; at meanwhile, it is connected with SIM900A via serial port and uploads data to the central server through GPRS. Test showed that the design functioned well with low packet error and had sound practical value.

ISCC 2015

18-19, December, 2015

Guangzhou, China

\footnotetext{
${ }^{1}$ Speaker

${ }^{2}$ This study is supported by Central Universities Fundamental Research Project (3142014126)
} 


\section{Introduction}

With the development of electrical control technology, it is necessary to study a kind of shear monitoring system, which is based on Internet and distributed modules[1]. At present, many domestic researchers have achieved some progress in the field of shear monitoring.

Liu Yunxiadesigned the shearer monitoring system based on the field bus technology to achieve the long-term acquisition transmission of shear state data[2] . Liu Jun designed a remote monitoring system of shear with PLC as the control core and put forward a scheme of system reliability from the control class distribution[3], redundancy mode and so on; Qiu Jinbo introduced a distributed shearer monitoring device based on DSP and can bus and made a brief description on the practical application effect[4]. Liu Ying designed a condition monitoring system of shearer based on wireless sensor network[5]; Ma Guoming analyzed the application of multi sensor information and information fusion technology in the condition monitoring, and made it realized on shear[6].

In the researches above, as the monitoring system is usually based on or partly based on the traditional wired data transmission, it still has some limitations in practical applications because of the special environment in coal mine, such as wiring difficulties.

Li Miaomiao designed a wireless data acquisition system based on ARM processor[7], but used the ARM processor as the core of the gateway node costs high; moreover, the maintenance of the equipment is difficult.

In order to solve the above problems, a ZigBee-GPRS communication scheme has been designed highlighting low cost, easy configuration and appropriateness to the coal mine; at the same time, in order to verify the stability of the monitoring system, the signal strength and the packet loss rate of the data received in the simulation have been carried out.

\section{Theoretical Analysis of System}

\subsection{General Structure of System}

According to the framework of ZigBee and GPRS network structure, the construction of coal mining machine state monitoring system is designed. The overall structure of the system is shown in Fig. 1:

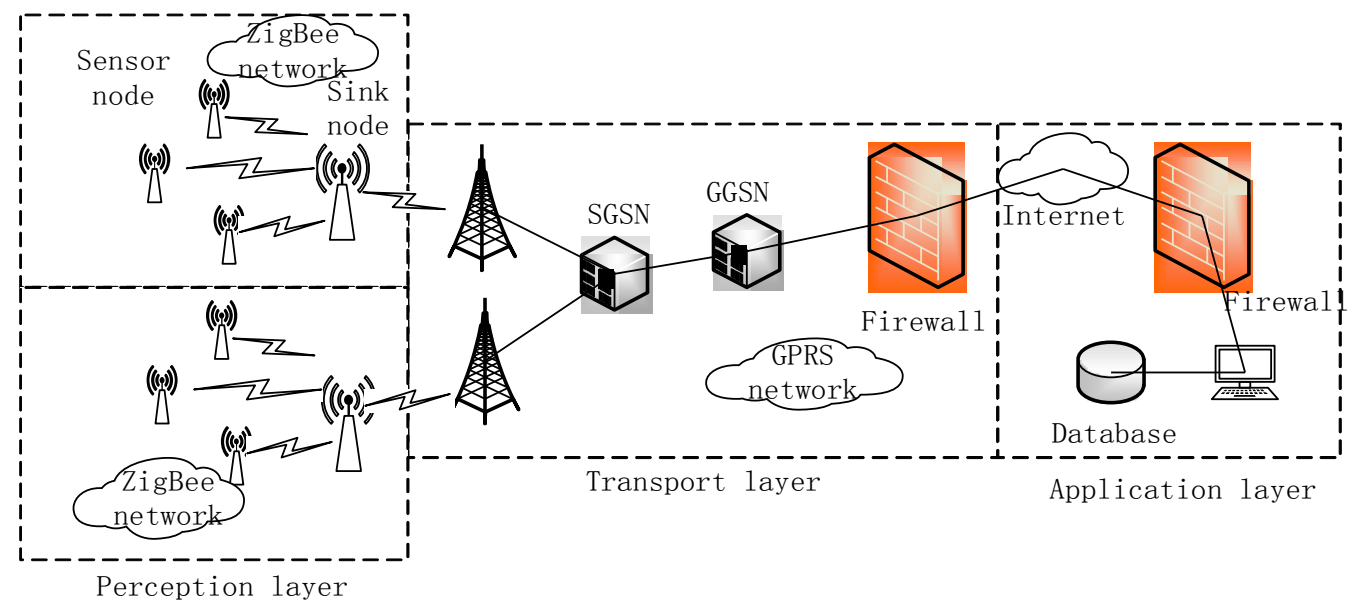

Figure 1: Overall Structure of the System

The system is composed of the ZigBee network, the remote data transmission GPRS network and the monitoring center. The node of ZigBee network is composed of the sensor module, the data processing module, the data transmission module and the power supply module. After the data which is processed by the sensor module analyzed by the processing 
module, it is transmitted to the gateway by the data transmission module, which will be sent to the monitoring center by GPRS network upon protocol conversion.

\subsection{Topology Structure Design of ZigBee Network}

According to the characteristics of shear and working face of the coal mining, the cluster network topology structure is chosen, like the root of the tree. The wireless network is shown in Fig. 2.

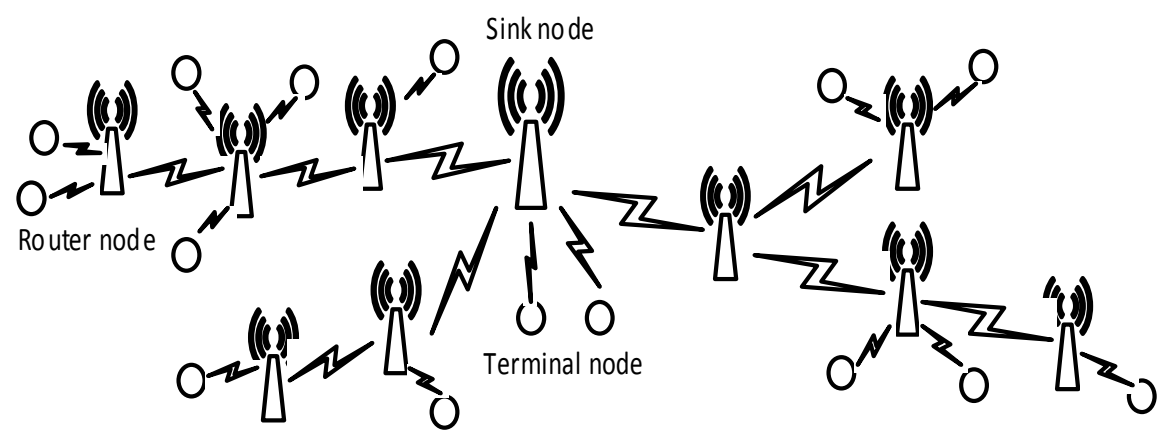

Figure 2: Topology Structure

\section{Design of State Monitoring Network of Shear}

\subsection{Hardware Design of ZigBee Network Node}

In design of the ZigBee network node, the most important part is the design of low power consumption. For hardware, low power consumption is decided by the choice of chip. ZigBee terminal node uses CC2530 as the core control module. The main peripheral modules are sensor modules (current sensor, voltage sensor, temperature sensor, etc.), wireless communication module, CC2591 and power supply module, as shown in Fig. 3.

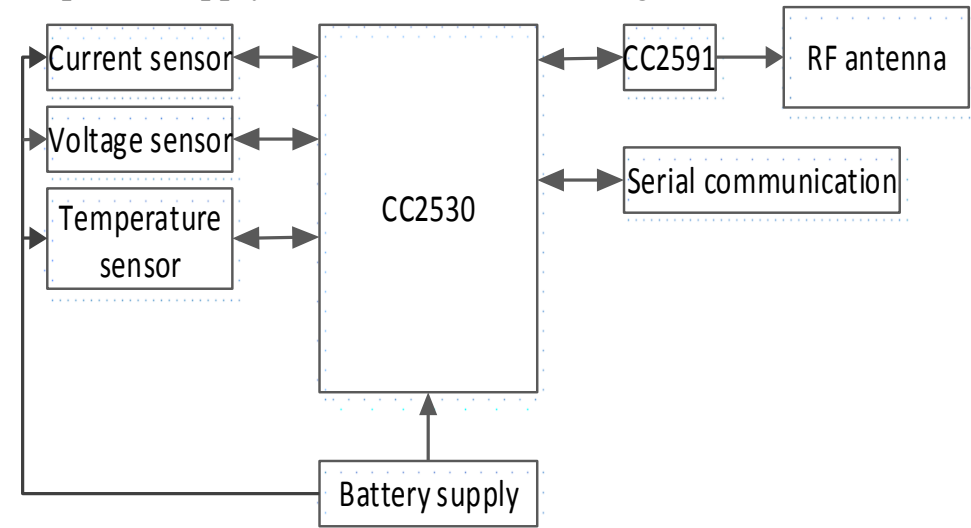

Figure 3: Design of Node

The application of CC2530 in the terminal node is shown in Fig. 4. 


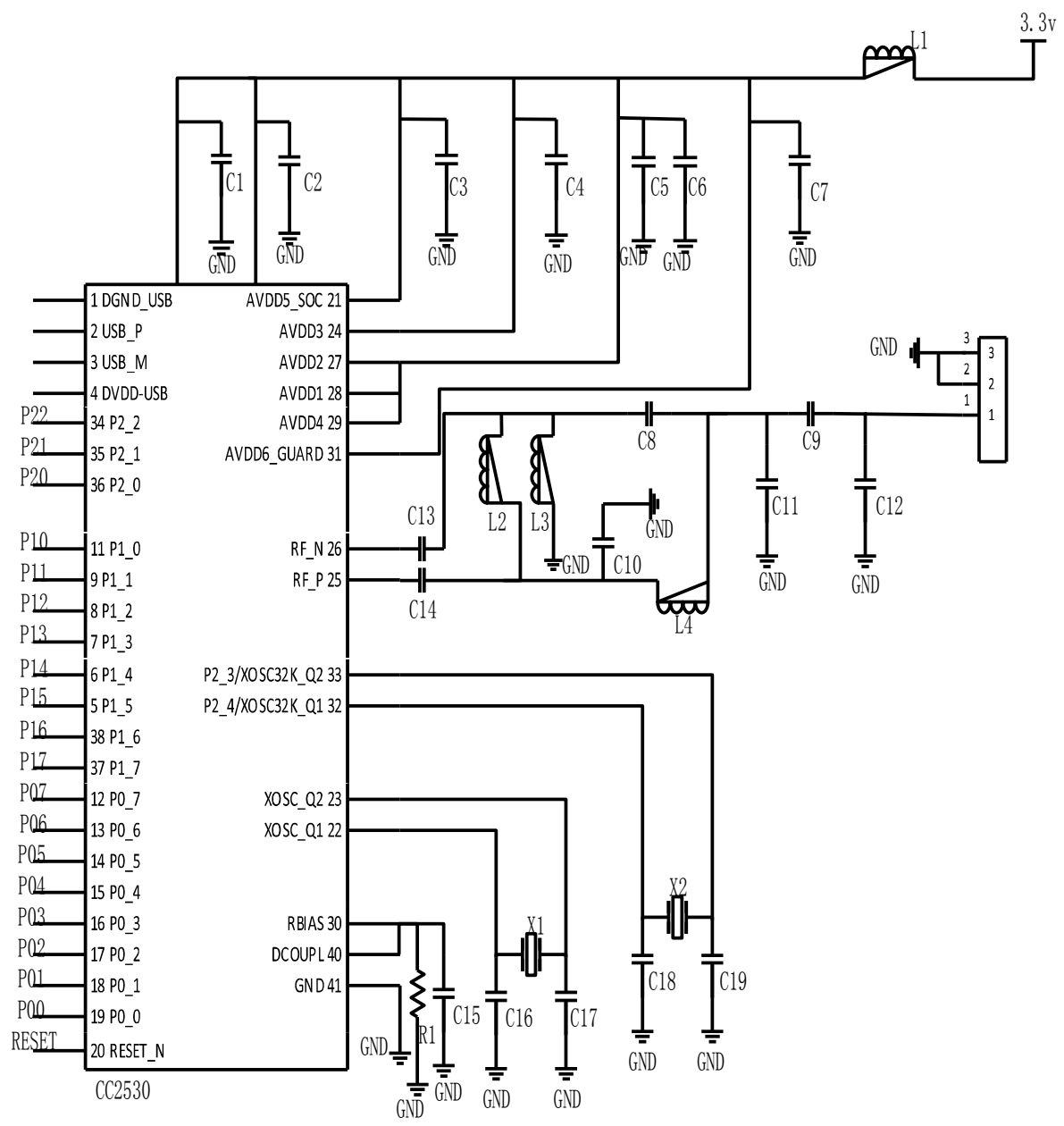

Figure 4: Application Circuit of CC2530

The hardware design of the router node is similar to that of the terminal node with only the peripheral sensor module removed.

\subsection{Hardware Design of ZigBee-GPRS Gateway}

\subsubsection{Gateway Processor Module}

Due to the special environment in coal mine, there is difficulty in the equipment maintenance; thus it is necessary to study a one-time configuration without the need to dynamically adjust the gateway node design.

In this research, the gateway node is designed by using CC2530. The ZigBee coordinator module is integrated into the central processor module. The application of CC2530 in the gateway node is shown in Fig. 5. 


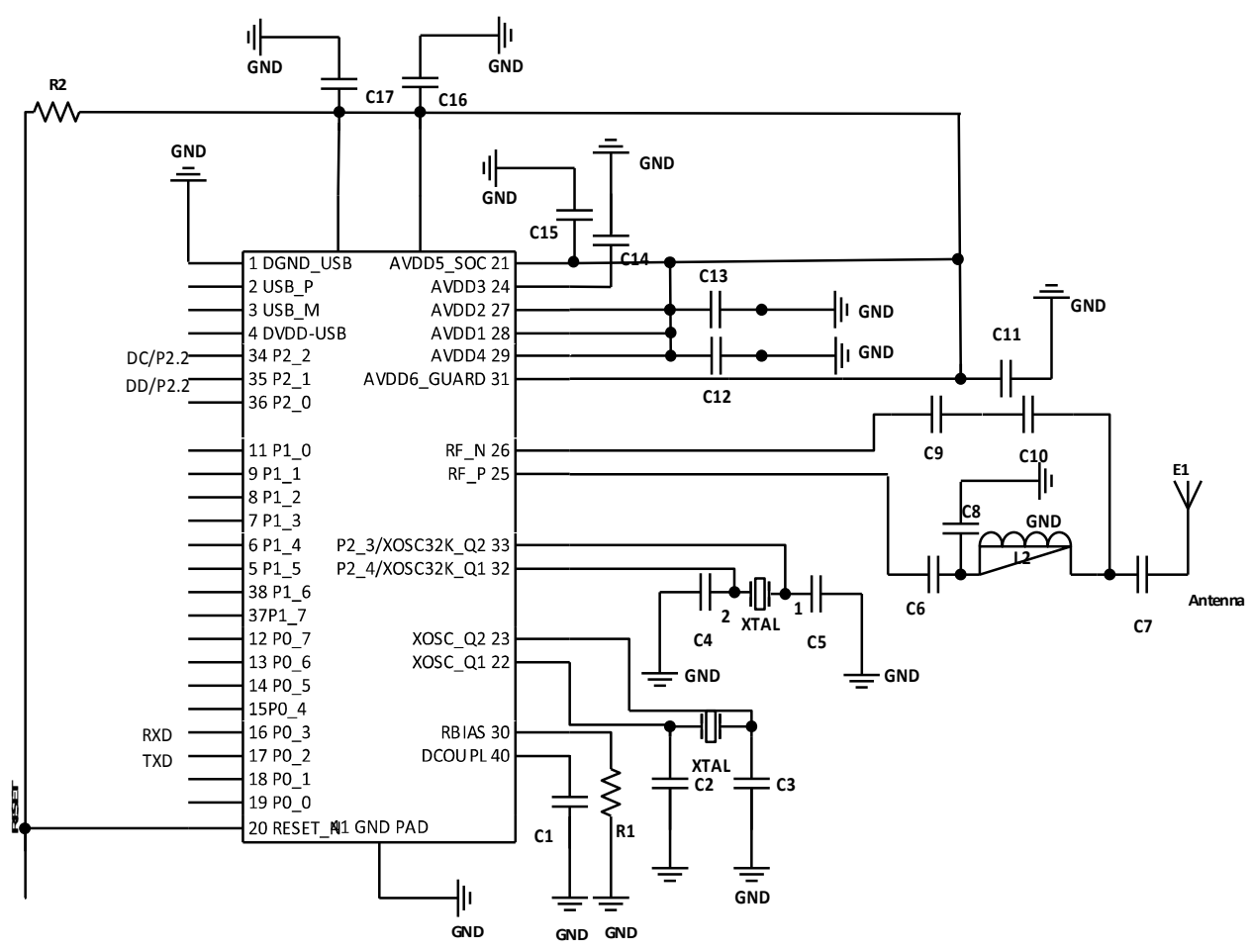

Figure 5: Application Circuit of CC2530

In Fig. 5, pin P1_4 is connected to the network status indicator, and the mode is the general I/O interface. After the gateway node is powered up, the indicator light flashes to indicate the success of the ZigBee network. Pin P0_2 and pin P0_3 are used as the TX USART0 port and RX port, the mode is the peripheral $\mathrm{I} / \mathrm{O}$ interface, connected to the serial port device GPRS module. P2_1 and P2_2 are used for debugging interface, through which, the users could access the simulator to debug the hardware.

\subsubsection{GPRS Module}

SIM900A is used in the GPRS module and the lowest power consumption can be reduced to $1.0 \mathrm{~mA}$, which is suitable for the low power consumption of coal mine environment. The circuit of the GPRS module is shown in Fig. 6.

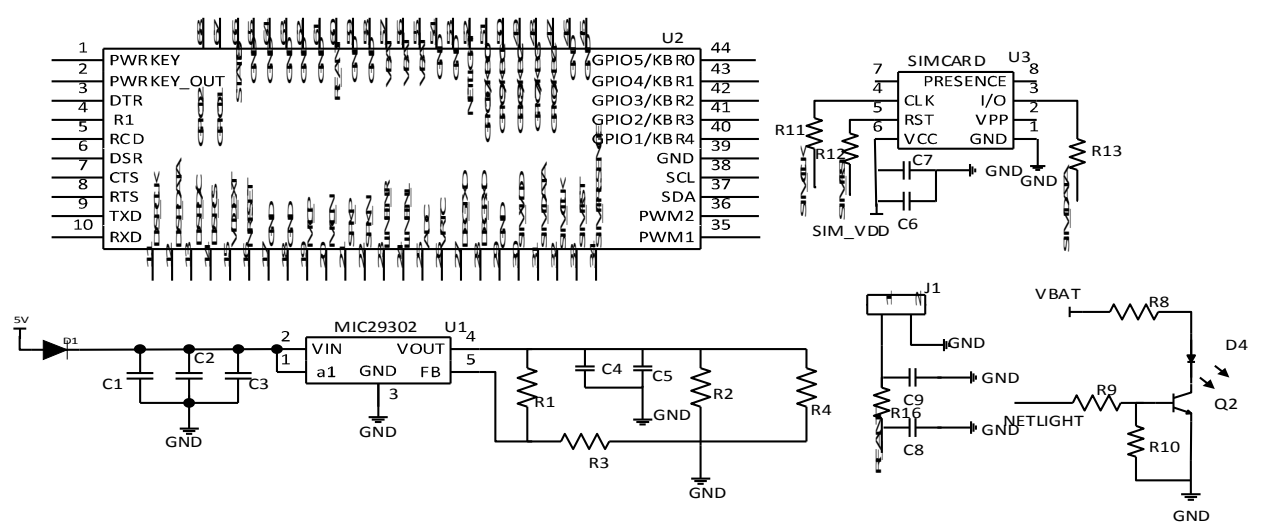

Figure 6: Circuit of the GPRS 
The GPRS module is composed of the antenna interface circuit, the card slot circuit, the serial circuit and the power supply circuit. R11, R12, R13 are connected to the SIM_RST, SIM_DATA, and SIM_CLK, as the matching impedance between SIM900A and SIM. NETLIGHT signal, as the driving signal, indicating whether the GPRS module is in the working state, whether it is registered to the network and whether the network connection is normal.

\subsection{Power Module}

The power module is responsible for the power supply of the nodes. As the system adopts wireless transmission, it is not easy to replace the battery frequently underground; so this system should use the low power design scheme and prolong the use time of the battery. The power supply voltage of the central processor module is $3.3 \mathrm{~V}$ and the power supply voltage of the GPRS module is $5 \mathrm{~V}$; therefore, $12 \mathrm{~V}$ lithium battery is used and converted to $5 \mathrm{~V}$ through LM7805, and then converted to $3.3 \mathrm{~V}$ output voltage through the LM117 voltage regulator chip.

\subsection{Monitoring Network Software Design}

The monitoring network software is developed on the platform integrated IAR EW. The software design process is shown in Fig. 7.

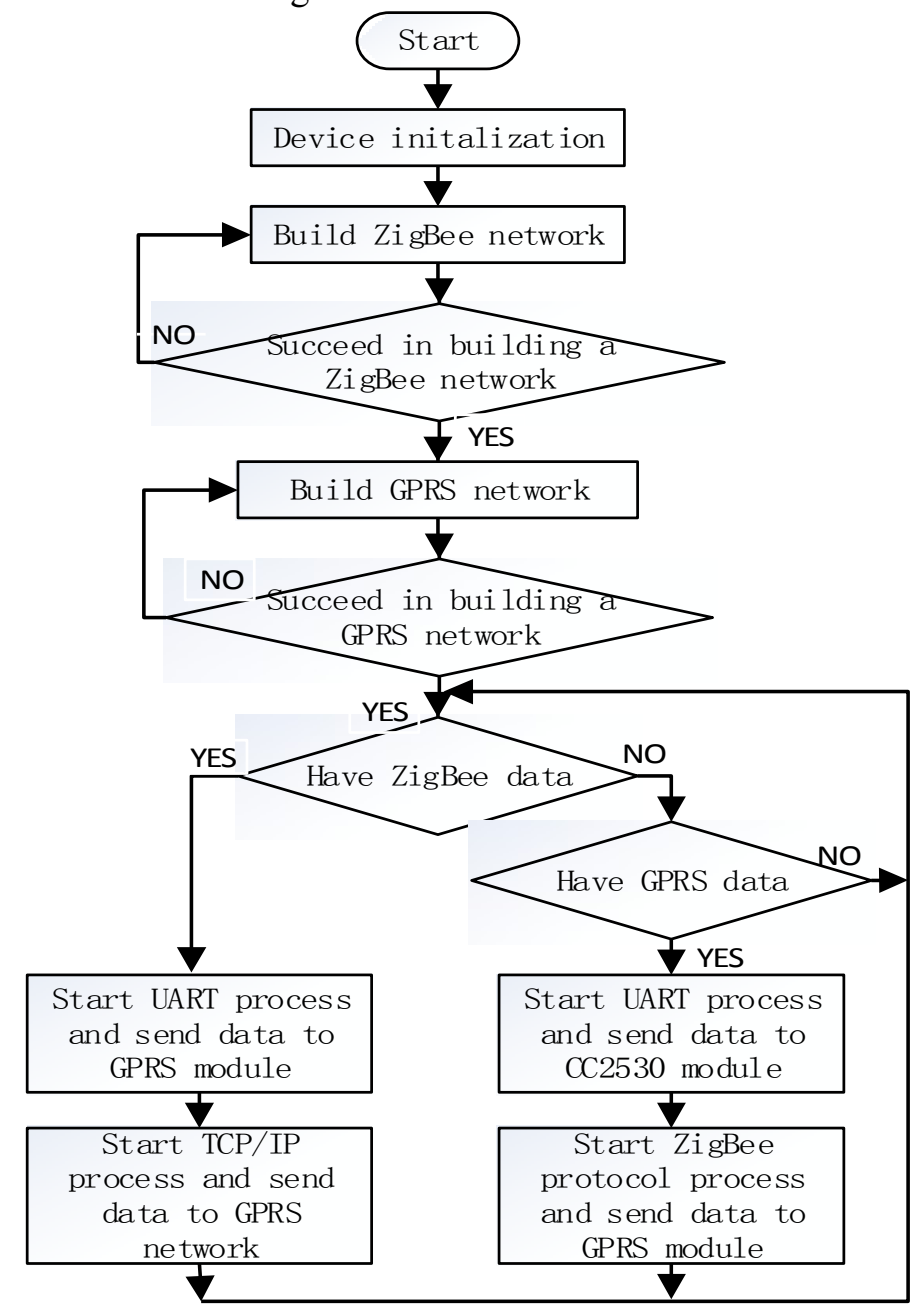

Figure 7: Software Design Process

After the network device is initialized, the ZigBee network and GPRS network are established by calling the API interface function and the primitive function to send AT commands to the SIM900A network through USART0. After the network is established successfully, the terminal nodes scan channel and the monitoring data are transmitted to the 
gateway through the best route, finally to the server through GPRS network. TasksArr[] stores the address of the task processing function. The storage of the tasks is in TasksEvents[]. The network performs data collection, forwarding and uploading. Program uses OSAL to complete the detection of the IDX cycle and ensures that the interrupt response is completed.

\section{Experiment}

This experiment is carried out in the laboratory under the condition of simulated tunnel, including 6 terminal nodes and 1 gateway node; and the network topology of each node is the same as that in Fig. 2. The signal strength RSSI and the packet loss rate of the node are shown in Table 1.

\begin{tabular}{llc}
\hline Distance $/ \mathrm{m}$ & $\mathrm{RSSI} / \mathrm{dBm}$ & Loss rate/\% \\
\hline 0 & -37.5 & 0 \\
5 & -60.3 & 0 \\
10 & -68.7 & 1 \\
15 & -72.0 & 0 \\
20 & -78.0 & 0 \\
25 & -80.5 & 2.4 \\
30 & -78.6 & 0 \\
35 & -75.9 & 0 \\
40 & -83.5 & 0 \\
45 & -85.0 & 2.5 \\
50 & -76.9 & 0 \\
55 & -83.7 & 0 \\
60 & -82.8 & 0 \\
70 & -87.2 & 3.2 \\
\hline
\end{tabular}

Table 1: Signal Strength RSSI and Packet Loss Rate

Results of the signal intensity variation with distance are shown in Fig. 8. After data fitting, $\mathrm{R}^{2}=0.9436$. Experiment shows that the $70 \mathrm{~m}$ packet loss rate is less than $10 \%, \mathrm{R}^{2}$ is close to 1 and the system has some practical value.

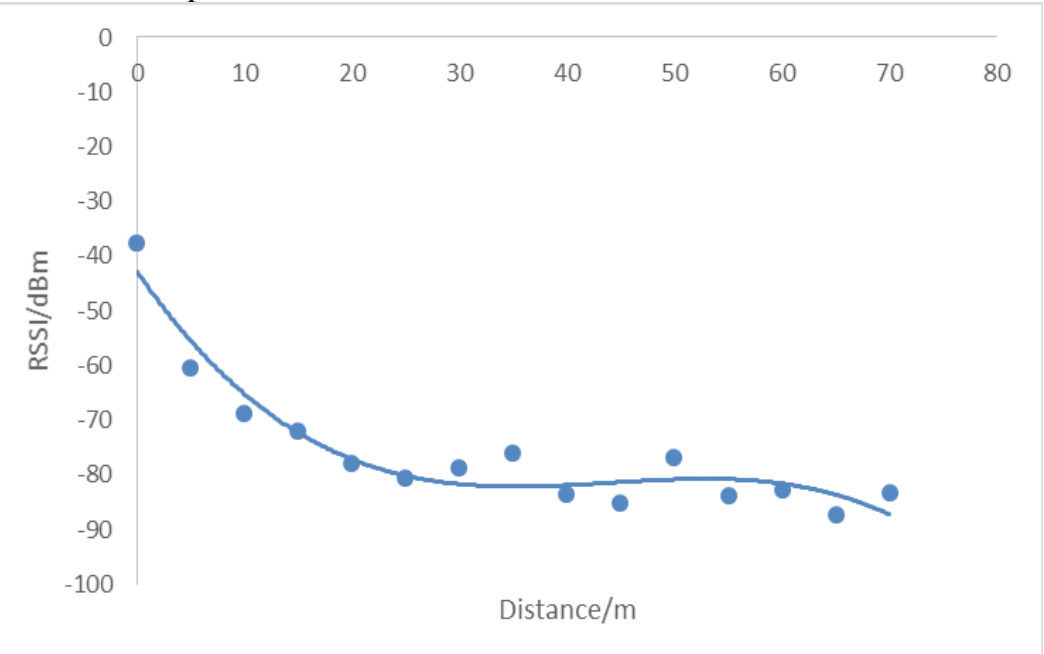

Figure 8: Signal Intensity Variation with Distance

\section{Conclusion}

In this study, the shear monitoring system supporting network convergence is introduced from the hardware design of the terminal node and the gateway node as well as the software communication process. Information of the shear was sent to the server through ZigBee network, ZigBee-GPRS gateway and GPRS network.

Experiments show that the network has low packet loss rate, reliable operation and good prospects for further development. 


\section{References}

[1] Shihong Zhang, The innovation of the technology of the fully mechanized coal mining machine in our country [J] Journal of coal, 2010, 35(11):1899-1902. (In Chinese)

[2] Yunxia Liu, Jingwen Huang, Dejian Tan. Monitoring and monitoring system of electric traction shearer based on field bus technology [J]. Industrial automation, 2002, (6):29-31. (In Chinese)

[3] Jun Liu, Zhongbin Wang, Ke Niu, et al. Research on Key Technologies of remote monitoring system of coal mining machine [J]. Coal science and technology, 2010, 38(6):67-69. (In Chinese)

[4] Jinbo Qiu, Zhenjian Liu.. Coal science and technology based on DSP and CAN bus[J], 2008, 36(3):128-134.(In Chinese)

[5] Ying Liu, Xiaoguang Zhang. Coal mining machine based on wireless sensor network and network query system [J]. Coal mine machinery, 2011, 32(7):261-263. (In Chinese)

[6] Qiang Sun, Mingguo Ma, Yupo Yang, et al. Application and analysis of wireless sensor information fusion in coal mining machine condition monitoring [J]. Coal mining machinery, 2014, (5):253-257. (In Chinese)

[7] Miaomiao Li, Liangguang Li, Zhiping Chen, et al. Design of wireless data acquisition system based on Zigbee and GPRS coal mine [J]. Coal mine machinery, 2012, 33(12):239-241. (In Chinese) 